PROCEEDINGS OF THE

AMERICAN MATHEMATICAL SOCIETY

Volume 137, Number 9, September 2009, Pages 3057-3066

S 0002-9939(09)09841-4

Article electronically published on February 16, 2009

\title{
THE S-TRANSFORM \\ OF SYMMETRIC PROBABILITY MEASURES WITH UNBOUNDED SUPPORTS
}

\author{
OCTAVIO ARIZMENDI E. AND VICTOR PÉREZ-ABREU
}

(Communicated by Richard C. Bradley)

\begin{abstract}
The Voiculescu $S$-transform is an analytic tool for computing free multiplicative convolutions of probability measures. It has been studied for probability measures with non-negative support and for probability measures having all moments and zero mean. We extend the $S$-transform to symmetric probability measures with unbounded support and without moments. As an application, a representation of symmetric free stable measures is derived as a multiplicative convolution of the semicircle measure with a positive free stable measure.
\end{abstract}

\section{INTRODUCTION}

Let $\mathcal{M}$ be the class of all Borel probability measures on the real line $\mathbb{R}$ and let $\mathcal{M}_{b}$ and $\mathcal{M}^{+}$be the subclasses of $\mathcal{M}$ consisting of probability measures with bounded support and of probability measures having support on $\mathbb{R}_{+}=[0, \infty)$, respectively. A measure $\mu$ in $\mathcal{M}$ is symmetric if $\mu(-B)=\mu(B)$ for all Borel sets $B$, it has all moments if $m_{k}(\mu)=\int_{\mathbb{R}}|t|^{k} \mu(\mathrm{d} t)<\infty$, for each integer $k \geq 1$, and has zero mean when $m_{1}(\mu)=0$.

The free multiplicative convolution $\otimes$ is a binary operation on $\mathcal{M}$ that was introduced by Voiculescu [15] to describe the multiplication of free (non-commuting) random variables. This operation is important in the study of free products of certain operator algebras [14] and has found one of its main applications in the theory of large dimensional random matrices, since it allows one to compute the asymptotic spectrum of the product of two independent random matrices from the individual asymptotic spectra ([8], [16]).

An important analytic tool for computing the free multiplicative convolution of two probability measures is the Voiculescu $S$-transform. It was introduced in [15] for non-zero mean distributions in $\mathcal{M}_{b}$ and further studied by Bercovici and Voiculescu 6] in the case of probability measures in $\mathcal{M}^{+}$with unbounded support; see also [5]. In particular, this $S$-transform is important for computing free multiplicative convolutions of positive free stable distributions with themselves, as shown in [3].

Received by the editors September 29, 2008, and, in revised form, November 18, 2008.

2000 Mathematics Subject Classification. Primary 46L54, 15 A52.

Key words and phrases. Multiplicative convolution, free stable distribution, random matrix.

The first author's research was supported by SNI-CONACYT Grant A. I. 4337 and the Statistics Laboratory of CIMAT.

(C)2009 American Mathematical Society Reverts to public domain 28 years from publication 
Recently, Raj Rao and Speicher [13] extended the $S$-transform to the case of measures in $\mathcal{M}$ having zero mean and all moments. Their main tools are combinatorial arguments based on moment calculations. This allows them to compute interesting free multiplicative convolutions of measures with bounded support, such as the one of the Marchenko-Pastur distribution with the semicircle distribution.

The purpose of this paper is to extend the $S$-transform to general symmetric probability measures on $\mathbb{R}$ with unbounded support and without moments. We follow an analytic approach and use the known results for the non-negative unbounded support case. This allows us to compute interesting examples of free multiplicative convolutions of probability measures in $\mathcal{M}^{+}$with general symmetric probability measures in $\mathcal{M}$. As an important example of distributions without finite moments and unbounded supports we consider the free stable distributions studied in [3, 6], 12. As an application, we show that any symmetric free stable distribution is the multiplicative convolution of the semicircle distribution with a positive free stable distribution. A reproducing property for these distributions is also found.

The paper is organized as follows. In Section 2 we review non-commutative random variables and their free products and collect known results on the $S$-transform that are used later on. Section 3 is dedicated to the study of the $S$-transform of symmetric probability measures on $\mathbb{R}$ as well as to the free multiplicative convolution of symmetric distributions in $\mathcal{M}$ with distributions in $\mathcal{M}^{+}$. Finally, Section 4 gives a description of the symmetric free stable distributions as the multiplicative convolution of the semicircle distribution with a positive free stable distribution.

\section{Preliminaries on free CONVOlutions}

Following [16, we recall that a non-commutative probability space $(\mathcal{A}, \varphi)$ is called a $W^{*}$-probability space if $\mathcal{A}$ is a non-commutative von Neumann algebra and $\varphi$ is a normal faithful trace. A family of unital von Neumann subalgebras $\left\{\mathcal{A}_{i}\right\}_{i \in I} \subset \mathcal{A}$ in a $W^{*}$-probability space is said to be free if $\varphi\left(a_{1} a_{2} \cdots a_{n}\right)=0$ whenever $\varphi\left(a_{j}\right)=0, a_{j} \in \mathcal{A}_{i_{j}}$, and $i_{1} \neq i_{2} \neq \ldots \neq i_{n}$. A self-adjoint operator $X$ is said to be affiliated with $\mathcal{A}$ if $f(X) \in \mathcal{A}$ for any bounded Borel function $f$ on $\mathbb{R}$. In this case it is also said that $X$ is a (non-commutative) random variable. Given a self-adjoint operator $X$ affiliated with $\mathcal{A}$, the distribution of $X$ is the unique measure $\mu_{X}$ in $\mathcal{M}$ satisfying

$$
\varphi(f(X))=\int_{\mathbb{R}} f(x) \mu_{X}(\mathrm{~d} x)
$$

for every Borel bounded function $f$ on $\mathbb{R}$. If $\left\{\mathcal{A}_{i}\right\}_{i \in I}$ is a family of free unital von Neumann subalgebras and $X_{i}$ is a random variable affiliated with $\mathcal{A}_{i}$ for each $i \in I$, then the random variables $\left\{X_{i}\right\}_{i \in I}$ are said to be free.

The Cauchy transform of a probability measure $\mu$ on $\mathbb{R}$ is defined, for $z \in \mathbb{C} \backslash \mathbb{R}$, by

$$
G_{\mu}(z)=\int_{\mathbb{R}} \frac{1}{z-x} \mu(\mathrm{d} x) .
$$

It is well known that $G_{\mu}$ is an analytic function in $\mathbb{C} \backslash \mathbb{R}, G_{\mu}: \mathbb{C}_{+} \rightarrow \mathbb{C}_{-}$and that $G_{\mu}$ determines uniquely the measure $\mu$. The reciprocal of the Cauchy transform is the function $F_{\mu}(z): \mathbb{C}_{+} \rightarrow \mathbb{C}_{+}$defined by $F_{\mu}(z)=1 / G_{\mu}(z)$. It was proved in [6] that there are positive numbers $\eta$ and $M$ such that $F_{\mu}$ has a right inverse $F_{\mu}^{-1}$ 
defined on the region

$$
\Gamma_{\eta, M}:=\{z \in \mathbb{C}|| \operatorname{Re}(z) \mid<\eta \operatorname{Im}(z), \quad \operatorname{Im}(z)>M\} .
$$

The Voiculescu transform of $\mu$ is defined by

$$
\phi_{\mu}(z)=F_{\mu}^{-1}(z)-z
$$

on any region of the form $\Gamma_{\eta, M}$, where $F_{\mu}^{-1}$ is defined; see [3, [6]. The free cumulant transform is a variant of $\phi_{\mu}$ defined as

$$
\mathcal{C}_{\mu}^{\boxplus}(z)=z \phi_{\mu}\left(\frac{1}{z}\right)=z F_{\mu}^{-1}\left(\frac{1}{z}\right)-1,
$$

for $z$ in a domain $D_{\mu} \subset \mathbb{C}_{-}$such that $1 / z \in \Gamma_{\eta, M}$ where $F_{\mu}^{-1}$ is defined; see [1].

The free additive convolution of two probability measures $\mu_{1}, \mu_{2}$ on $\mathbb{R}$ is defined as the probability measure $\mu_{1} \boxplus \mu_{2}$ on $\mathbb{R}$ such that $\phi_{\mu_{1} \boxplus \mu_{2}}(z)=\phi_{\mu_{1}}(z)+\phi_{\mu_{2}}(z)$ or equivalently

$$
\mathcal{C}_{\mu_{1} \boxplus \mu_{2}}^{\boxplus}(z)=\mathcal{C}_{\mu_{1}}^{\boxplus}(z)+\mathcal{C}_{\mu_{2}}^{\boxplus}(z)
$$

for $z \in D_{\mu_{1}} \cap D_{\mu_{2}}$. It turns out that $\mu_{1} \boxplus \mu_{2}$ is the distribution of the sum $X_{1}+X_{2}$ of two free random variables $X_{1}$ and $X_{2}$ having distributions $\mu_{1}$ and $\mu_{2}$ respectively.

On the other hand, the free multiplicative operation $\nabla$ on $\mathcal{M}$ is defined as follows; see [6. Let $\mu_{1}, \mu_{2}$ be probability measures on $\mathbb{R}$, with $\mu_{1} \in \mathcal{M}^{+}$and let $X_{1}, X_{2}$ be free random variables such that $\mu_{X_{i}}=\mu_{i}$. Since $\mu_{1}$ is supported on $\mathbb{R}_{+}, X_{1}$ is a positive self-adjoint operator and $\mu_{X_{1}^{1 / 2}}$ is uniquely determined by $\mu_{1}$. Hence the distribution $\mu_{X_{1}^{1 / 2} X_{2} X_{1}^{1 / 2}}$ of the self-adjoint operator $X_{1}^{1 / 2} X_{2} X_{1}^{1 / 2}$ is determined by $\mu_{1}$ and $\mu_{2}$. This measure is called the free multiplicative convolution of $\mu_{1}$ and $\mu_{2}$ and it is denoted by $\mu_{1} \otimes \mu_{2}$. This operation on $\mathcal{M}$ is associative and commutative.

The next result was proved in $\left[\underline{6}\right.$ for probability measures in $\mathcal{M}^{+}$with unbounded support.

Proposition 1. Let $\mu \in \mathcal{M}^{+}$such that $\mu(\{0\})<1$. The function

$$
\Psi_{\mu}(z)=\int_{0}^{\infty} \frac{z x}{1-z x} \mu(\mathrm{d} x), \quad z \in \mathbb{C} \backslash \mathbb{R}_{+}
$$

is univalent in the left-plane $i \mathbb{C}_{+}$and $\Psi_{\mu}\left(i \mathbb{C}_{+}\right)$is a region contained in the circle with diameter $(\mu(\{0\})-1,0)$. Moreover, $\Psi_{\mu}\left(i \mathbb{C}_{+}\right) \cap \mathbb{R}=(\mu(\{0\})-1,0)$.

Let $\chi_{\mu}: \Psi_{\mu}\left(i \mathbb{C}_{+}\right) \rightarrow i \mathbb{C}_{+}$be the inverse function of $\Psi_{\mu}$. The $S$-transform of $\mu$ is the function

$$
S_{\mu}(z)=\chi(z) \frac{1+z}{z}
$$

The following result shows the role of the $S$-transform as an analytic tool for computing free multiplicative convolutions. It was shown in 14 for measures in $\mathcal{M}^{+}$ with bounded support and in [6] for measures in $\mathcal{M}^{+}$with unbounded support.

We collect Corollaries 6.6 and 6.7 and Lemma 6.9 in [6] as follows.

Proposition 2. Let $\mu_{1}$ and $\mu_{2}$ be probability measures in $\mathcal{M}^{+}$with $\mu_{i} \neq \delta_{0}, i=1,2$. Then $\mu_{1} \otimes \mu_{2} \neq \delta_{0}$ and

$$
S_{\mu_{1} \boxplus \mu_{2}}(z)=S_{\mu_{1}}(z) S_{\mu_{2}}(z)
$$

in that component of the common domain which contains $(-\varepsilon, 0)$ for small $\varepsilon>0$. Moreover, $\left(\mu_{1} \otimes \mu_{2}\right)(\{0\})=\max \left\{\mu_{1}(\{0\}), \mu_{2}(\{0\})\right\}$. 
Proposition 3. Let $\left\{\mu_{n}\right\}_{n=1}^{\infty}$ and $\left\{\nu_{n}\right\}_{n=1}^{\infty}$ be sequences of probability measures in $\mathcal{M}^{+}$converging to probability measures $\mu$ and $\nu$ in $\mathcal{M}^{+}$, respectively, in the weak* topology and such that $\mu \neq \delta_{0} \neq \nu$. Then, the sequence $\left\{\mu_{n} \otimes \nu_{n}\right\}_{n=1}^{\infty}$ converges to $\mu \otimes \nu$ in the weak ${ }^{*}$ topology.

The next proposition is a particular case of a recent result proved in [13] for probability measures $\mu_{1}, \mu_{2}$ on $\mathbb{R}$ with all moments, when $\mu_{1}$ has zero mean and $\mu_{2} \in \mathcal{M}^{+}$.

Proposition 4. Let $\mu_{1}$ be a compactly supported symmetric probability measure on $\mathbb{R}$ and let $\mu_{2} \in \mathcal{M}^{+}$have compact support, with $\mu_{i} \neq \delta_{0}, i=1,2$. Then, $\mu_{1} \otimes$ $\mu_{2} \neq \delta_{0}$ and

$$
S_{\mu_{1} \bowtie \mu_{2}}(z)=S_{\mu_{1}}(z) S_{\mu_{2}}(z) .
$$

From (44) and the fact that $\Psi_{\mu}(z)=\frac{1}{z} G_{\mu}\left(\frac{1}{z}\right)-1$, one obtains the following relation observed in [9] between the free cumulant transform and the $S$-transform:

$$
z=\mathcal{C}_{\mu}^{\boxplus}\left(z S_{\mu}(z)\right)
$$

This equation holds for measures in $\mathcal{M}^{+}$or in $\mathcal{M}_{b}$ with zero mean. It was suggested in [13] that (7) may be used to define $S$-transforms of general probability measures on $\mathbb{R}$.

We finally mention that for free random variables having distributions with compact supports, a combinatorial approach to multiplicative convolutions is given in [11 and a Banach algebra approach to the $S$-transform is presented in 7 .

\section{MAIN RESUlts}

For a general probability measure $\mu$ on $\mathbb{R}$, let

$$
\Psi_{\mu}(z)=\int_{\mathbb{R}} \frac{z t}{1-z t} \mu(\mathrm{d} t)=\frac{1}{z} G_{\mu}\left(\frac{1}{z}\right)-1, \quad z \in \mathbb{C} \backslash \mathbb{R}_{+} .
$$

The function $\Psi_{\mu}$ determines the measure $\mu$ uniquely since the Cauchy transform $G_{\mu}$ does.

Given a symmetric probability measure $\mu$ on $\mathbb{R}$, let $Q(\mu)=\mu^{2}$ be the probability measure in $\mathcal{M}^{+}$induced by the map $t \rightarrow t^{2}$. The transformation $Q$ has been used, for example, in 10 to study a relation between multiplicative convolutions of positively supported probability measures with the commutator and the anticommutator of two symmetric probability measures. Likewise, the transformation $\mu^{2}$ is called in [2] the push-forward of the measure $\mu$, and it is used to study the so-called rectangular free convolution.

We first prove two important relations between the Cauchy transforms and the $\Psi$ functions of $\mu$ and $\mu^{2}$.

Proposition 5. Let $\mu$ be a symmetric probability measure $\mu$ on $\mathbb{R}$. Then

a) $G_{\mu}(z)=z G_{\mu^{2}}\left(z^{2}\right), z \in \mathbb{C} \backslash \mathbb{R}_{+}$.

b) $\Psi_{\mu}(z)=\Psi_{\mu^{2}}\left(z^{2}\right), z \in \mathbb{C} \backslash \mathbb{R}_{+}$. 
Proof. a) Use the symmetry of $\mu$ twice to obtain

$$
\begin{aligned}
G_{\mu}(z) & =\int_{\mathbb{R}} \frac{1}{z-t} \mu(\mathrm{d} t)=\int_{\mathbb{R}_{+}} \frac{1}{z-t} \mu(\mathrm{d} t)+\int_{\mathbb{R}_{+}} \frac{1}{z+t} \mu(\mathrm{d} t) \\
& =2 z \int_{\mathbb{R}_{+}} \frac{1}{z^{2}-t^{2}} \mu(\mathrm{d} t)=z \int_{\mathbb{R}^{2}} \frac{1}{z^{2}-t^{2}} \mu(\mathrm{d} t) \\
& =z \int_{\mathbb{R}_{+}} \frac{1}{z^{2}-t} \mu^{2}(\mathrm{~d} t)=z G_{\mu^{2}}\left(z^{2}\right) .
\end{aligned}
$$

b) Use (8) twice and (a) to obtain

$$
\begin{aligned}
\Psi_{\mu}(z) & =\int_{\mathbb{R}} \frac{z x}{1-z x} \mu(\mathrm{d} x)=\frac{1}{z} G_{\mu}\left(\frac{1}{z}\right)-1 \\
& =\frac{1}{z^{2}} G_{\mu^{2}}\left(\frac{1}{z^{2}}\right)-1=\Psi_{\mu^{2}}\left(z^{2}\right),
\end{aligned}
$$

which shows (b).

Theorem 6. Let $\mu$ be a symmetric probability measure $\mu$ on $\mathbb{R}$.

a) If $\mu \neq \delta_{0}$, the function $\Psi_{\mu}$ is univalent in each of the following two disjoint sets:

$$
\begin{array}{ll}
H=\left\{z \in \mathbb{C}_{+} \mid\right. & |\operatorname{Re}(z)|<\operatorname{Im}(z)\}, \\
\widetilde{H}=\left\{z \in \mathbb{C}_{-} \mid\right. & |\operatorname{Re}(z)|<|\operatorname{Im}(z)|\} .
\end{array}
$$

Therefore $\Psi_{\mu}$ has a unique inverse $\chi_{\mu}: \Psi_{\mu}(H) \rightarrow H$ and a unique inverse $\widetilde{\chi}_{\mu}$ : $\Psi_{\mu}(\widetilde{H}) \rightarrow \widetilde{H}$.

b) If $\mu \neq \delta_{0}$, the $S$-transforms

$$
S_{\mu}(z)=\chi_{\mu}(z) \frac{1+z}{z} \text { and } \widetilde{S}_{\mu}(z)=\widetilde{\chi}_{\mu}(z) \frac{1+z}{z}
$$

are such that

$$
S_{\mu}^{2}(z)=\frac{1+z}{z} S_{\mu^{2}}(z) \text { and } \widetilde{S}_{\mu}^{2}(z)=\frac{1+z}{z} S_{\mu^{2}}(z)
$$

for $z$ in $\Psi_{\mu}(H)$ and $\Psi_{\mu}(\widetilde{H})$ respectively.

Proof. a) Let $h: \mathbb{C} \rightarrow \mathbb{C}$ be the function $h(z)=z^{2}$. Then $h(H)=h(\widetilde{H})=i \mathbb{C}_{+}$and therefore $h$ is univalent in $H$ and in $\widetilde{H}$. On the other hand, since $\mu^{2} \in \mathcal{M}^{+}$, by Proposition 1, $\Psi_{\mu^{2}}(z)$ is univalent in $i \mathbb{C}_{+}$and therefore $\Psi_{\mu^{2}}\left(z^{2}\right)$ is univalent in $H$ and in $\widetilde{H}$.

b) Since $\mu^{2} \in \mathcal{M}^{+}$, from Proposition 1, the unique inverse $\chi_{\mu^{2}}$ of $\Psi_{\mu^{2}}$ is such that $\chi_{\mu^{2}}: \Psi_{\mu^{2}}\left(i \mathbb{C}_{+}\right) \rightarrow i \mathbb{C}_{+}$. Thus, use (a) to obtain $\Psi_{\mu^{2}}\left(\chi_{\mu}^{2}(z)\right)=\Psi_{\mu}\left(\chi_{\mu}(z)\right)=z$ for $z \in \Psi_{\mu}(H)$ and the uniqueness of $\chi_{\mu^{2}}$ gives $\chi_{\mu^{2}}(z)=\chi_{\mu}^{2}(z), z \in \Psi_{\mu}(H)$. Hence

$$
\begin{aligned}
S_{\mu}^{2}(z) & =\chi_{\mu}^{2}(z)\left(\frac{1+z}{z}\right)^{2}=\chi_{\mu^{2}}(z)\left(\frac{1+z}{z}\right)^{2} \\
& =S_{\mu^{2}}(z) \frac{1+z}{z}, \quad z \in \Psi_{\mu}(H),
\end{aligned}
$$

and similarly for $\widetilde{S}_{\mu}(z)$. 
The following is the main result of this paper. It shows how to compute the multiplicative convolution of a symmetric probability measure on $\mathbb{R}$ with a probability measure on $\mathbb{R}_{+}$. No existence of moments or bounded supports for the probability measures are assumed.

Theorem 7. Let $\mu$ and $\nu$ be probability measures on $\mathbb{R}$ such that $\mu$ is symmetric, $\nu \in \mathcal{M}^{+}$and $\mu \neq \delta_{0} \neq \nu$. Let $S_{\mu}$ and $\widetilde{S}_{\mu}$ be the two $S$-transforms of $\mu$. Then

$$
S_{\mu 凶 \nu}(z)=S_{\mu}(z) S_{\nu}(z) \text { and } \widetilde{S}_{\mu 凶 \nu}(z)=\widetilde{S}_{\mu}(z) S_{\nu}(z)
$$

are the two $S$-transforms of the symmetric probability measure $\mu \otimes \nu$, where the functions in (11) are considered in the common domain which contains $(-\varepsilon, 0)$ for small $\varepsilon>0$.

The key to proving this theorem is the following lemma, which is a result of independent interest.

Lemma 8. Let $\mu$ and $\nu$ be probability measures on $\mathbb{R}$ such that $\mu$ is symmetric, $\nu \in \mathcal{M}^{+}$and $\mu \neq \delta_{0} \neq \nu$. Then

$$
\nu \otimes \mu^{2} \otimes \nu=(\mu \otimes \nu)^{2} .
$$

Proof. We first prove the result when $\mu$ and $\nu$ have compact supports. In this case, since $\mu$ has zero mean, use Proposition 4 to obtain $S_{\mu \otimes \nu}(z)=S_{\mu}(z) S_{\nu}(z)$. Then, since $\mu \otimes \nu$ is a symmetric probability measure, the use of (10) gives

$$
\frac{z}{z+1} S_{\mu 凶 \nu}^{2}(z)=S_{(\mu \otimes \nu)^{2}}(z)
$$

Hence, use (10) again to obtain

$$
S_{(\mu \otimes \nu)^{2}}(z)=\frac{z}{z+1} S_{\mu}^{2}(z) S_{\nu}^{2}(z)=S_{\mu^{2}}(z) S_{\nu}^{2}(z) .
$$

Since $\mu^{2}$ and $\nu$ are in $\mathcal{M}^{+}$, by Proposition $2 . S_{\mu^{2}}(z) S_{\nu}^{2}(z)=S_{\nu \otimes^{2} \otimes_{\nu}}(z)$, and therefore (12) holds for $\mu$ and $\nu$ having compact supports.

Next, for $\mu$ and $\nu$ with unbounded supports, choose symmetric probability measures $\mu_{n}$ on $\mathbb{R}$ with compact support and $\nu_{n}$ in $\mathcal{M}^{+}$also with compact support, $n \geq 1$, such that $\left\{\mu_{n}\right\}_{n=1}^{\infty}$ and $\left\{\nu_{n}\right\}_{n=1}^{\infty}$ converge to $\mu$ and $\nu$, respectively, in the weak* topology. From the first part of the proof we obtain $S_{\nu_{n} \otimes \mu_{n}^{2} \otimes \nu_{n}}=S_{\left(\mu_{n} \otimes \nu_{n}\right)^{2}}$, $n \geq 1$. On the other hand, since $\mu_{n}^{2}, \nu_{n}$ and $\left(\mu_{n} \otimes \nu_{n}\right)^{2}$ belong to $\mathcal{M}^{+}$, by Proposition 3. the sequences $\left\{\nu_{n} \otimes \mu_{n}^{2} \otimes \nu_{n}\right\}_{n=1}^{\infty}$ and $\left\{\left(\mu_{n} \otimes \nu_{n}\right)^{2}\right\}_{n=1}^{\infty}$ converge to $\nu \otimes \mu^{2} \otimes \nu$ and $(\mu \otimes \nu)^{2}$, respectively, in the weak* topology. This proves the result.

Proof of Theorem 7 . We obtain the result as a consequence of Theorem 6 (b) and the above lemma as follows:

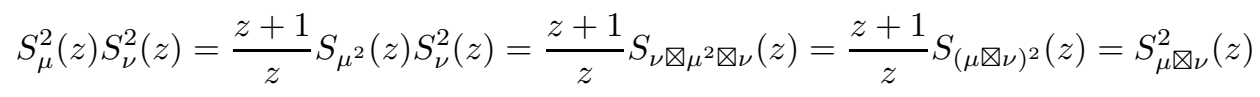
for $z \in \Psi_{\mu}(H) \cap \Psi_{\nu}(H)$ and similarly for $\widetilde{S}_{\mu}$.

We finally observe that (17) also holds for symmetric probability measures on $\mathbb{R}$.

Proposition 9. Let $\mu$ be a symmetric probability measure on $\mathbb{R}$. Then

$$
z=\mathcal{C}_{\mu}^{\boxplus}\left(z S_{\mu}(z)\right) \text { and } z=\mathcal{C}_{\mu}^{\boxplus}\left(z \widetilde{S}_{\mu}(z)\right)
$$

whenever $1 /\left[z S_{\mu}(z)\right]$ and $1 /\left[z \widetilde{S}_{\mu}(z)\right]$ belong to domains $\Gamma_{\eta, M}$ where $F_{\mu}^{-1}$ is defined, respectively. 
Proof. Let $\chi_{\mu}$ be the inverse of $\Psi$ in $H$. Using (8) we have that for $z \in \Psi_{\mu}(H)$, $z=\Psi_{\mu}\left(\chi_{\mu}(z)\right)=G_{\mu}\left(1 / \chi_{\mu}(z)\right) / \chi_{\mu}(z)-1$. Thus, $G_{\mu}\left(1 / \chi_{\mu}(z)\right)=(1+z) \chi_{\mu}(z)$ and

$$
F_{\mu}\left(\frac{1}{\chi_{\mu}(z)}\right)=\frac{1}{(1+z) \chi_{\mu}(z)} \text {. }
$$

Then,

$$
\frac{1}{\chi_{\mu}(z)}=F_{\mu}^{-1}\left(\frac{1}{(1+z) \chi_{\mu}(z)}\right) .
$$

Finally, use (4) to obtain

$$
z=(1+z) \chi_{\mu}(z) F_{\mu}^{-1}\left(\frac{1}{(1+z) \chi_{\mu}(z)}\right)-1=\mathcal{C}_{\mu}^{\boxplus}\left(z S_{\mu}(z)\right)
$$

and similarly for $\widetilde{S}_{\mu}$.

\section{Representation of Symmetric Free stable laWs}

Following [1, it is said that a probability measure $\mu$ on $\mathbb{R}$ is stable with respect to the free additive convolution $\boxplus$ defined by (5), or simply free stable or $\boxplus$-stable, if the class

$$
\{\psi(\mu): \psi \text { is an increasing affine transformation }\}
$$

is closed under the free additive convolution $\boxplus$. We denote by $S^{\boxplus}(\mathbb{R})$ the class of all free stable distributions on $\mathbb{R}$. They are examples of probability measures on $\mathbb{R}$ with unbounded supports, without all moments and without atoms. These distributions and their domains of attraction have been studied in [3], [5] and [12]. Here we are interested in symmetric free stable distributions $S_{s}^{\boxplus}(\mathbb{R})$ and in free stable distributions $S^{\boxplus}\left(\mathbb{R}_{+}\right)$with non-negative support.

We first use the results in the Appendix in 3, to find the free cumulant transforms of distributions in $S_{s}^{\boxplus}(\mathbb{R})$ and $S^{\boxplus}\left(\mathbb{R}_{+}\right)$.

Lemma 10. a) A probability measure $\sigma$ on $\mathbb{R}_{+}$belongs to $S^{\boxplus}\left(\mathbb{R}_{+}\right)$if and only if there exist $\alpha, 0<\alpha<1, \theta>0$ and $\gamma_{0} \geq 0$ such that

$$
\mathcal{C}_{\sigma}^{\boxplus}(z)=\gamma_{0} z-\theta e^{i \alpha \pi} z^{\alpha} .
$$

b) A probability measure $\nu$ on $\mathbb{R}_{+}$belongs to $S_{s}^{\boxplus}(\mathbb{R})$ if and only if there exist $\alpha$, $0<\alpha<2$ and $\theta>0$ such that when $1 \leq \alpha \leq 2$,

$$
\mathcal{C}_{\nu}^{\boxplus}(z)=\theta e^{i(\alpha-2) \frac{\pi}{2}} z^{\alpha},
$$

and when $0<\alpha<1$,

$$
\mathcal{C}_{\nu}^{\boxplus}(z)=-\theta e^{i \alpha \frac{\pi}{2}} z^{\alpha} .
$$

Proof. We use the relation $\mathcal{C}_{\nu}^{\boxplus}(z)=z \phi_{\nu}\left(\frac{1}{z}\right)$ given by (4) between the free cumulant transform $\mathcal{C}_{\mu}^{\boxplus}$ and the Voiculescu transform $\phi_{\mu}$ defined by (3).

a) From Section A4 and (4) in the Appendix in [3] and Theorem 7.5 in [6], $\sigma$ is a free stable distribution with support in $(0, \infty)$ if and only if there exist $\alpha$, $0<\alpha<1$, and $\theta>0$ such that $\phi_{\sigma}(z)=-\theta e^{i \alpha \pi} z^{-\alpha+1}$. Then, use (4) to obtain (14).

b) Similarly, from (2) in the Appendix of [3] and Theorem 7.5 in [6], the measure $\mu$ is a symmetric free stable distribution (with asymmetry coefficient $\rho=1$ in the notation of [3]) if and only if there exist $\alpha, 0<\alpha \leq 2$, and $\theta>0$, such 
that $\phi_{\nu}(z)=\theta e^{i(\alpha-2) \frac{\pi}{2}} z^{-\alpha+1}$ when $1 \leq \alpha \leq 2$ and $\phi_{\nu}(z)=-e^{i \alpha \frac{\pi}{2}} z^{-\alpha+1}$ when $0<\alpha<1$. In both cases, (b) follows by using (44).

For $0<\alpha<1$, we denote by $\sigma_{\alpha}$ an element in $S^{\boxplus}\left(\mathbb{R}_{+}\right)$with free cumulant transform (14) and drift $\gamma_{0}=0$. In this case $\sigma_{\alpha}$ has support $(0, \infty)$, and we say that $\sigma_{\alpha}$ is a positive free $\alpha$-stable distribution. In general, a distribution in $S^{\boxplus}\left(\mathbb{R}_{+}\right)$ has support $\left(\gamma_{0}, \infty\right)$, in which case we use the notation $\sigma_{\alpha, \gamma_{0}}$. Similarly, $\nu_{\alpha}$ is a symmetric free $\alpha$-stable distribution, $0<\alpha<2$, if $\nu_{\alpha} \in S^{\boxplus}(\mathbb{R})$ and its free cumulant transform is given by (15) or (16). In what follows the power functions $z^{c}$ are defined through their principal branches in $\mathbb{C}_{+}$.

The $S$-transforms of positive and symmetric free $\alpha$-stable distributions are as follows.

Proposition 11. a) If $\sigma_{\alpha}$ is a positive free $\alpha$-stable distribution, $0<\alpha<1$, then there exists $\theta_{\alpha}>0$ such that

$$
S_{\sigma_{\alpha}}(z)=\theta_{\alpha} e^{i(1-\alpha) \frac{\pi}{\alpha}} z^{\frac{1-\alpha}{\alpha}} .
$$

b) If $\nu_{\alpha}$ is a symmetric free $\alpha$-stable distribution, $0<\alpha \leq 2$, then there exists $\theta_{\alpha}>0$ such that

$$
S_{\nu_{\alpha}}(z)=\theta_{\alpha} e^{i(2-\alpha) \frac{\pi}{2 \alpha}} z^{\frac{1-\alpha}{\alpha}}
$$

Proof. a) Use (71) and (14) to obtain the equation

$$
z=\mathcal{C}_{\sigma_{\alpha}}^{\boxplus}\left(z S_{\sigma_{\alpha}}(z)\right)=-\theta e^{i \alpha \pi}\left(z S_{\sigma_{\alpha}}(z)\right)^{\alpha}=\theta e^{i(1+\alpha) \pi} z^{\alpha} S_{\sigma_{\alpha}}^{\alpha}(z),
$$

from which (17) follows for $z \in \mathbb{C}_{+}$, since $\phi_{\sigma_{\alpha}}$ is analytic on $\mathbb{C}_{+}$.

b) Let $0<\alpha<1$. Use (13) and (16) to obtain the equation

$$
z=\mathcal{C}_{\nu_{\alpha}}^{\boxplus}\left(z S_{\nu_{\alpha}}(z)\right)=-\theta e^{i \alpha \frac{\pi}{2}}\left(z S_{\nu_{\alpha}}(z)\right)^{\alpha}=\theta e^{i\left(1+\frac{\alpha}{2}\right) \pi} z^{\alpha} S_{\nu_{\alpha}}^{\alpha}(z),
$$

from which (18) follows for $z \in \mathbb{C}_{+}$, since $\phi_{\nu_{\alpha}}$ is analytic on $\mathbb{C}_{+}$. A similar result holds for $1 \leq \alpha \leq 2$.

The following result gives a representation of symmetric free stable distributions as the multiplicative convolution of the Wigner distribution with a positive free stable distribution. Recall that the standard semicircle or Wigner distribution is the probability measure

$$
w(\mathrm{~d} x)=\frac{1}{2 \pi} \sqrt{4-x^{2}} \cdot 1_{[-2,2]} \mathrm{d} x .
$$

It is a symmetric free stable distribution with $\alpha=2$ and therefore

$$
S_{w}(z)=\frac{1}{\sqrt{z}} .
$$

Theorem 12. For $0<\alpha<2$, the symmetric free $\alpha$-stable distribution $\nu_{\alpha}$ has the representation

$$
\nu_{\alpha}=\sigma_{\beta} \otimes w
$$

where $\sigma_{\beta}$ is a positive free $\beta$-stable distribution with index $\beta=2 \alpha /(2+\alpha)$.

Proof. Use (20), (17) and (18) to obtain

$$
S_{\nu_{\beta}}(z) S_{w}(z)=\theta_{\beta} e^{i(1-\beta) \frac{\pi}{\beta}} z^{\frac{1-\beta}{\beta}-\frac{1}{2}}=\theta_{\alpha} e^{i(2-\alpha) \frac{\pi}{2 \alpha}} z^{\frac{1-\alpha}{\alpha}}=S_{\nu_{\alpha}}(z) .
$$

Hence, Theorem 7 gives the result. 
For positive stable distributions, the following reproducing property holds 3 , Proposition A 4.3]: for all $s, t>0$,

$$
\sigma_{\frac{1}{1+s+t}}=\sigma_{\frac{1}{1+s}} \bigotimes \sigma_{\frac{1}{1+t}} .
$$

As a final result, we obtain a similar reproducing property for symmetric free stable distributions.

Proposition 13. For all $s, t>0$,

$$
\nu_{\frac{1}{1+s+t}}=\sigma_{\frac{1}{1+s}} \otimes \nu_{\frac{1}{1+t}}=\sigma_{\frac{1}{1+t}} \otimes \nu_{\frac{1}{1+s}} .
$$

Proof. This follows by using (17), (18) and Theorem 12 .

\section{ACKNOWLEDGMENTS}

The authors would like to thank Florent Benaych-Georges and Noriyoshi Sakuma for valuable comments on a preliminary version of this work.

\section{REFERENCES}

[1] O. E. Barndorff-Nielsen and S. Thorbjørnsen, Classical and Free Infinite Divisibility and Lévy Processes. In U. Franz and M. Schürmann (Eds.): Quantum Independent Increment Processes II. Quantum Lévy Processes, Classical Probability and Applications to Physics, Lecture Notes in Math. 1866, pp. 33-159, Springer, 2006. MR2213448 (2007h:60043)

[2] F. Benaych-Georges, On a surprising relation between the Marchenko-Pastur law, rectangular and square free convolutions, arXiv: 0808.3938v1 [math.PR], 28 Aug 2008.

[3] H. Bercovici and V. Pata, with an appendix by P. Biane, Stable laws and domains of attraction in free probability theory, Ann. of Math. (2) 149 (1999), 1023-1060. MR 1709310 (2000i:46061)

[4] H. Bercovici and V. Pata, A free analogue of Hinčin's characterization of infinite divisibility, Proc. Amer. Math. Soc. 128 (2000), 1011-1015. MR1636930 (2000i:46060)

5] H. Bercovici and D. Voiculescu, Lévy-Hinčin type theorems for multiplicative and additive free convolution, Pacific J. Math. 153 (1992), 217-248. MR.1151559 (93k:46052)

[6] H. Bercovici and D. Voiculescu, Free convolution of measures with unbounded support, Indiana Univ. Math. J. 42 (1993), 733-773. MR.1254116 (95c:46109)

[7] U. Haagerup, On Voiculescu's $R$ - and $S$-transforms for free non-commuting random variables. In D. Voiculescu (Ed): Free Probability Theory, Fields Institute Communications 12, pp. 127148, Amer. Math. Soc., Providence, RI, 1997. MR1426838 (98c:46137)

[8] F. Hiai and D. Petz, The Semicircle Law, Free Random Variables and Entropy, Mathematical Surveys and Monographs 77, Amer. Math. Soc., Providence, RI, 2000. MR 1746976 (2001j:46099)

[9] A. Nica and R. Speicher, A "Fourier transform" for multiplicative functions on non-crossing partitions, J. Algebraic Combin. 6 (1997), 141-160. MR1436532 (98i:46070)

[10] A. Nica and R. Speicher, Commutators of free random variables, Duke Math. J. 92 (1998), 553-592. MR 1620518 (99d:46084)

[11] A. Nica and R. Speicher, Lectures on the Combinatorics of Free Probability, London Mathematical Society Lecture Note Series 335, Cambridge University Press, Cambridge, 2006. MR.2266879 (2008k:46198)

[12] V. Pata, Lévy type characterization of stable laws for free random variables, Trans. Amer. Math. Soc. 347 (1995), 2457-2472. MR1311913 (96b:46091)

[13] N. Raj Rao and R. Speicher, Multiplication of free random variables and the $S$-transform: The case of vanishing mean, Elect. Comm. Probab. 12 (2007), 248-258. MR2335895|(2008f:46082)

[14] D. Voiculescu, Dual algebraic structures on operator algebras related to free products, J. Operator Theory 17 (1987), 85-98. MR873463(88c:46080) 
[15] D. Voiculescu, Multiplication of certain noncommuting random variables, J. Operator Theory 18 (1987), 223-235. MR915507 (89b:46076)

[16] D. Voiculescu, K. Dykema and A. Nica, Free Random Variables, CRM Monograph Series 1, Amer. Math. Soc., Providence, RI, 1992. MR1217253 (94c:46133)

School of Mathematics, University of Guanajuato, Valenciana, Guanajuato, Gto 36240, MEXICO

E-mail address: octavius@cimat.mx

Research Center for Mathematics, Cimat, Apartado Postal 402, Guanajuato, GtO 36000, MEXICO

E-mail address: pabreu@cimat.mx 